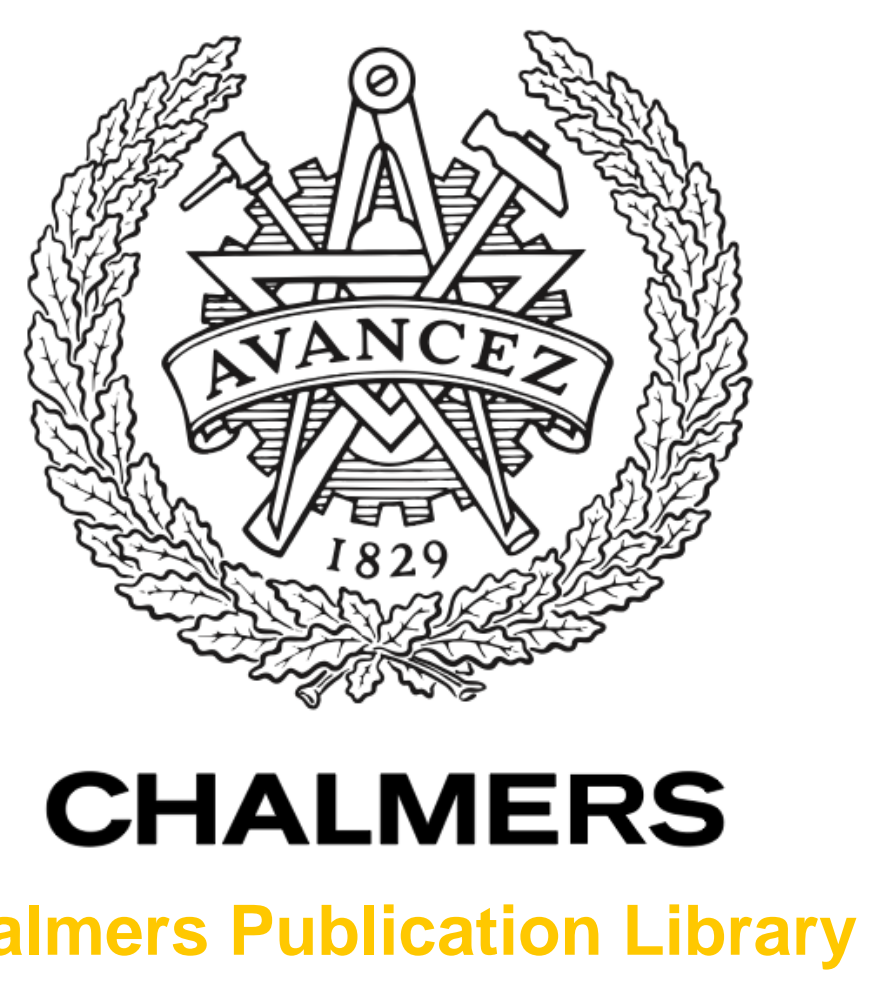

Chalmers Publication Library

\author{
Experimental Study of Frequency Multiplication in a Distributed Array of SIS \\ Junctions
}

This document has been downloaded from Chalmers Publication Library $(\mathrm{CPL})$. It is the author's version of a work that was accepted for publication in:

IEEE Transactions on Terahertz Science and Technology (ISSN: 2156-342X)

Citation for the published paper:

Billade, B. ; Pavolotsky, A. ; Belitsky, V. (2014) "Experimental Study of Frequency

Multiplication in a Distributed Array of SIS Junctions". IEEE Transactions on Terahertz

Science and Technology, vol. 4(2), pp. 254-259.

http://dx.doi.org/10.1109/TTHZ.2014.2304138

Downloaded from: http://publications.lib.chalmers.se/publication/194893

Notice: Changes introduced as a result of publishing processes such as copy-editing and formatting may not be reflected in this document. For a definitive version of this work, please refer to the published source. Please note that access to the published version might require a subscription.

Chalmers Publication Library (CPL) offers the possibility of retrieving research publications produced at Chalmers University of Technology. It covers all types of publications: articles, dissertations, licentiate theses, masters theses, conference papers, reports etc. Since 2006 it is the official tool for Chalmers official publication statistics. To ensure that Chalmers research results are disseminated as widely as possible, an Open Access Policy has been adopted.

The CPL service is administrated and maintained by Chalmers Library. 


\title{
Experimental study of frequency multiplication in a distributed array of SIS junctions
}

\author{
Bhushan Billade, Alexey Pavolotsky and Victor Belitsky, Senior Member, IEEE.
}

\begin{abstract}
We report the first experimental off-chip detection of frequency multiplication in a distributed array of Superconductor-Insulator-Superconductor (SIS) junctions. A test device consisting of series array of sixty eight $\mathrm{Nb} / \mathrm{Al}-\mathrm{AlO} \mathrm{O}_{x} / \mathrm{Nb}$ tunnel junctions was designed to study generation of the second harmonic in $182-192 \mathbf{G H z}$ band. The SIS array was exited with microwave radiation at $3 \mathrm{~mm}$ band using a quasi-optically coupled Gunn oscillator, and the output response of the device was studied using a double sideband SIS mixer operating in $163-211$ GHz range with $4-8 \mathbf{G H z}$ IF bandwidth.

We measured extremely sharp spectral signals, associated with the $\times 2$ frequency multiplication by the SIS array. Distinct single and multi-photon processes were observed in the SIS tunnel junction response to the applied microwave radiation, confirming device operation in quantum mode. The output power of the multiplied signal increases linearly with the power of the pumping signal up to certain level and them saturates. In attempt to verify that the device produces noticeable power, the output of the test device was connected to the LO port of the SIS mixer and an increase of $10-20 \%$ in the SIS mixer dark current was observed. Further development of the demonstrated principle of frequency multiplication, may lead to a practical frequency multiplier device.
\end{abstract}

Index Terms-Superconducting devices, frequency multiplier, thin-film circuits.

\section{INTRODUCTION}

$\mathbf{T}$ HE ability of a Superconductor-Insulator-Superconductor (SIS) tunnel junction to respond to a single absorbed photon and extremely sharp nonlinearity in their currentvoltage (I-V) characteristics, due to quasi-particle tunneling, has been utilized in ultra-low noise millimeter wave receivers [1]. These ultra-sensitive receivers generally use SIS mixers pumped with fundamental local oscillator (LO) frequency. However, there has been some work in sub-harmonically pumped SIS mixers using internally produced LO harmonic for mixing, either using series array [2] or using stacked SIS junction array [3].

Generally in these ultra-sensitive SIS systems, the LO source and frequency multipliers are placed at room temperature, and the LO signal is coupled to the mixer either using long stainless steel waveguide or quasi-optics. In case of waveguide LO coupling, most of the LO power is lost due to waveguide losses. There has been lot of work on frequency generation based on Josephson effect, both using low-Tc

Manuscript received on December xx, 20xx

Bhushan Billade is with the Onsala space observatory, Chalmers University of Technology, Gothenburg, Sweden. (e-mail: bhushan.billade@chalmers.se)

Alexey Pavolotsky and Victor Belitsky are with the Group of Advanced Receiver Development (GARD) at Chalmers University of Technology, Gothenburg, Sweden. superconductors [4] and using high-Tc superconductors [5]. Often the local oscillator source and the mixer are integrated on single chip [4], [6].

However, harmonic generation in SIS device using quasiparticle tunneling effect has never been experimentally demonstrated before. The extremely nonlinear behavior and quantum nature of operation of an SIS tunnel junction, give reasons to believe that an SIS device designed for frequency multiplication can operate with very high conversion efficiencies. Furthermore, most sensitive heterodyne receivers in the $\mathrm{mm} / \mathrm{sub}-\mathrm{mm}$ region use SIS mixers, which requires very low LO power. From this perspective, the SIS frequency multiplier is completely compatible with the system with respect to their operating environment and power requirements.

Possibility of using this nonlinear property of an SIS junction for frequency multiplication was suggested earlier [7], but there has not been any experimental effort to investigate this possibility further. One of the reasons could be that the power generated by such a multiplier device using single SIS junction would be very small. However, the total output power from a SIS multiplier device can be increased by increasing the junction area or by using an array of SIS junctions [8]. Keeping the same overall $R_{n}$ as a single junction, an array of $N$ junctions would have $N^{2}$ times higher power handling capacity.

Such a multiplier device can be easily arranged in a power combining configuration to boost the total output power further, and can also be integrated with the SIS mixer on the same chip, similar to the approach described for flux flow oscillators in [6]. In multi-pixel heterodyne systems, where complexity of the LO distribution system and LO power requirement would be one of the biggest challenges, an SIS frequency multiplier could offer very attractive alternative. In such scenario, the phase locking and distribution of the LO signal can be carried out at much lower frequencies.

In this paper we describe the first experimental investigation of SIS device as frequency multiplier. Even though the approach of integrating the multiplier and SIS mixer on single chip is very appealing, for the first demonstration we opt for a non-integrated approach.

\section{EXPERIMENTAL SIS MULTIPLIER}

We recognize that in principle the SIS junction as a frequency multiplier could operate in different modes. One can think of purely classical, classical-quantum, quantumclassical, and purely quantum mode, depending on the value of pumping and product frequencies and their relation with 
the superconducting gap value. A purely classical scenario would be when both the input and the output frequencies are less than the voltage nonlinearity $\left(\hbar \omega / e<\delta V_{\text {gap }}\right)$. In a classical-quantum mode, the input pumping frequency does not produce quantum response $\left(\hbar \omega / e<\delta V_{\text {gap }}\right)$ whereas, the output frequency is in the quantum regime. For a quantumclassical mode, one can think of a scenario, where the output frequency exceeds twice the gap frequency $\left(\hbar \omega / e>2 V_{\text {gap }}\right)$. In this paper we investigate a quantum-quantum mode, where both the pumping and the output frequencies produce quantum response $\left(2 V_{\text {gap }}>\hbar \omega / e>\delta V_{\text {gap }}\right)$.

The quantum theory of mixing [9] describes in great details the mechanism of tunneling current at different harmonics. The tunneling current through the SIS junction as a response to the applied pumping signal $\left(V_{\omega}\right)$ is represented as [9],

$$
I_{L O}(t)=a_{0}+\sum_{m=1}^{\infty}\left(2 a_{m} \cos m \omega t+2 b_{m} \sin m \omega t\right)
$$

where, the coefficients $a_{m}$ and $b_{m}$ are given by,

$$
\begin{aligned}
2 a_{m}= & \sum_{\substack{n=-\infty\\
}}^{\infty} J_{n}(\alpha)\left[J_{n+m}(\alpha)+V_{0}+n \hbar \omega / e\right) \\
2 b_{m}= & \sum_{n=-\infty}^{\infty} J_{n}(\alpha)\left[J_{n+m}(\alpha)-J_{n-m}(\alpha)\right] \\
& \times I_{K K}\left(V_{0}+n \hbar \omega / e\right)
\end{aligned}
$$

where, $\alpha=e V_{\omega} / \hbar \omega$ is the pumping factor, $J$ is the Bessel function, $a_{0}$ is the DC component of the current, and $I_{K K}$ Kramers-Kronig transform of the DC current-voltage characteristics of the tunnel junction.

In practical SIS mixer designs, if the geometrical capacitance of the SIS junction is large enough, higher harmonics $(\mathrm{m}>1)$ are usually ignored. Whereas, in the cases where the capacitance of the junction is small, higher harmonics would no longer be shorted by the intrinsic junction capacitance or the embedding circuitry, and thus could be extracted out of the system. The power level of these higher harmonics across the SIS junction is just few nano-Watts/ $\mu m^{2}$ [9]. Consequently, a bigger area junction or an array of the SIS junctions should boost the available output power of the harmonics.

\section{Multiplier Chip Design}

A test device with Superconductor-InsulatorSuperconductor Multiplier Array (SuMA) was designed and fabricated as shown in Fig. 1. Aiming for enhancing the generated power of the harmonic signal, we decided to use both, bigger size SIS junctions, and an array of 68 junctions. The SuMA chip was designed for frequency multiplication factor of two $(\times 2)$ with a WR-10 input $(3 \mathrm{~mm}$ band), resulting in the frequency output in $1.5 \mathrm{~mm}$ band. The reason for choosing this particular frequency band, was the availability of the $163-211 \mathrm{GHz}$ SIS mixer [10], that we intended to use to study the generated signals.

The SuMA chip uses an array of 68 SIS tunnel junctions as shown in Fig. 1. The chip consists of the SIS junction array, two filter sections, waveguide probes for pumping

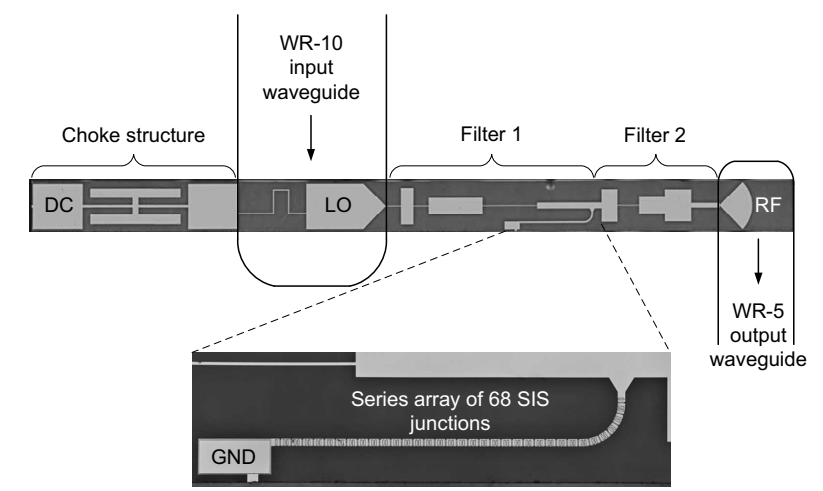

Fig. 1. Photograph of the test chip showing the series array of 68 SIS junctions, two filter sections, RF and LO probes, position of the input and the output waveguides, and DC bias and ground (GND) ports. Each junction is $25 \mu \mathrm{m}^{2}$ in area with the overall chip dimensions of $415 \mu \mathrm{m} \times 6700 \mu \mathrm{m}$.

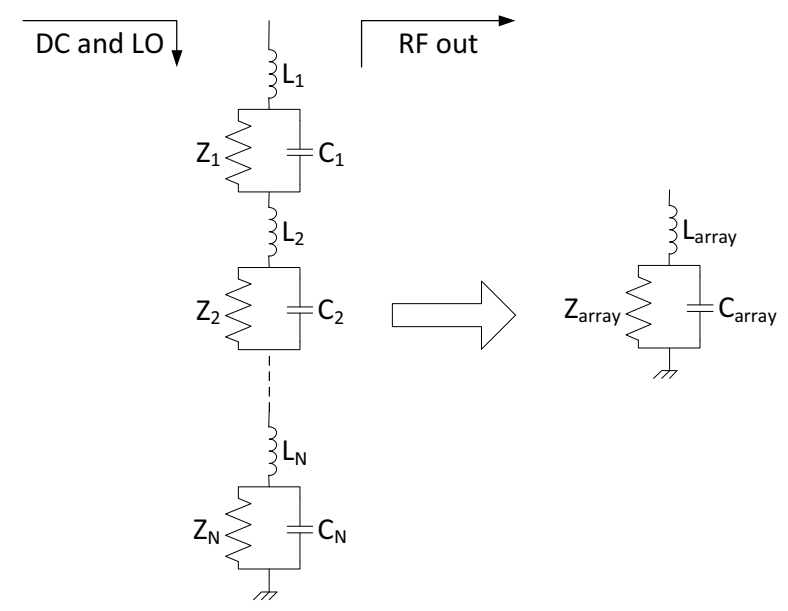

Fig. 2. Simplified equivalent circuit of the multiplier.

and multiplied signal, and DC contact pads. The chip was fabricated on a 90 um thick crystal Quartz substrate using inhouse $\mathrm{Nb} / \mathrm{Al}-\mathrm{AlO} \mathrm{O}_{x} / \mathrm{Nb}$ thin-film technology [11]. Each of the 68 junctions has $25 \mu \mathrm{m}^{2}$ area, and an $R_{n} A$ value of $50 \Omega \mu m^{2}$.

Fig. 2 shows an equivalent circuit of the experimental multiplier. Each individual SIS junction was modeled as an impedance in parallel with capacitance, similar to the approach described in [12], [10]. The individual junctions are connected together using a short piece of transmission line. Since this short piece of the connecting line is on a $90 \mu \mathrm{m}$ thick substrate, it becomes highly inductive. Therefore, the array of 68 junctions connected with the inductive lines become electrically long, and must be considered distributed. The value of the parallel capacitance was determined by using approach described in [13]. The value of the parallel impedance $Z$ was determined using the Tucker's theory [9],

$$
Z_{m m^{\prime}}=1 / Y_{m m^{\prime}}=1 /\left(G_{m m^{\prime}}+i \cdot B_{m m^{\prime}}\right)
$$

where, 


$$
\begin{aligned}
G_{m m^{\prime}} & =\frac{e}{2 \hbar \omega_{m^{\prime}}} \sum_{n, n^{\prime}=-\infty}^{\infty} J_{n}(\alpha) J_{n^{\prime}}(\alpha) \delta_{m-m^{\prime}, n-n^{\prime}} \\
& \times\left\{I_{d c}\left(V_{0}+\frac{n^{\prime} \hbar \omega}{e}+\frac{\hbar \omega_{m^{\prime}}}{e}\right)-I_{d c}\left(V_{0}+\frac{n^{\prime} \hbar \omega}{e}\right)\right. \\
& \left.+I_{d c}\left(V_{0}+\frac{n \hbar \omega}{e}\right)-I_{d c}\left(V_{0}+\frac{n \hbar \omega}{e}-\frac{\hbar \omega_{m^{\prime}}}{e}\right)\right\}
\end{aligned}
$$

and

$$
\begin{aligned}
B_{m m^{\prime}} & =\frac{e}{2 \hbar \omega_{m^{\prime}}} \sum_{n, n^{\prime}=-\infty}^{\infty} J_{n}(\alpha) J_{n^{\prime}}(\alpha) \delta_{m-m^{\prime}, n-n^{\prime}} \\
& \times\left\{I_{K K}\left(V_{0}+\frac{n^{\prime} \hbar \omega}{e}+\frac{\hbar \omega_{m^{\prime}}}{e}\right)-I_{K K}\left(V_{0}+\frac{n^{\prime} \hbar \omega}{e}\right)\right. \\
& \left.+I_{K K}\left(V_{0}+\frac{n \hbar \omega}{e}\right)-I_{K K}\left(V_{0}+\frac{n \hbar \omega}{e}-\frac{\hbar \omega_{m^{\prime}}}{e}\right)\right\},
\end{aligned}
$$

At the pumping frequency the impedance of the junction is $Y_{11}=Y_{-1-1}$, whereas, at the output frequency the impedance is determined using the element $Y_{22}=Y_{-2-2}$.

Since the SIS array represents two different impedances to the embedding network, one at the pumping frequency $\left(Y_{11}\right)$ and another at the output $(\times 2)$ frequency $\left(Y_{22}\right)$, the overall optimization was carried out using two separate $S$-parameter simulations. All the components were simulated separately using full 3D EM solver [14], and were combined in ADS circuit simulator [15] to verify the overall response. Two filter sections as shown in Fig. 1 were used, to isolate the input and the output frequency components from each other.

Fig. 3 shows a picture of the multiplier waveguide block with an E-plane split. The SuMA chip is placed inside a chip channel and the coupling between the microwave radiation in waveguide mode and the chip is achieved using probes extending inside the waveguides. For the high frequency side a radial probe as described in [16] and [17] was used. Whereas, for the low frequency side, a probe with integrated bias-T [18] was used to allow the DC biasing of the SIS array. An SMA connector was used for DC biasing the array, where, the center pin of the SMA was connected to the DC port of the chip using bond wires. The chip was DC grounded by connecting bond wires from the ground port (GND in Fig. 1) directly to the waveguide block.

\section{Measurement And Results}

The characterization of the device performance was carried out at $4 \mathrm{~K}$ in a cryostat with Helium closed cycle refrigerator. Fig. 4, shows the test setup used for the measurements of the SuMA. To investigate the response from distributed SIS array, we use a double sideband SIS mixer [10] that covers the RF frequencies from $163-211 \mathrm{GHz}$ with 4 to $8 \mathrm{GHz}$ intermediate frequency (IF) bandwidth, connected at the output of SuMA. The SIS array was pumped using a quasi-optically coupled W-band $(83-105 \mathrm{GHz})$ Gunn oscillator and the output from the SIS array was coupled to the input of the mixer using a short piece of waveguide. In the experiment, the Josephson

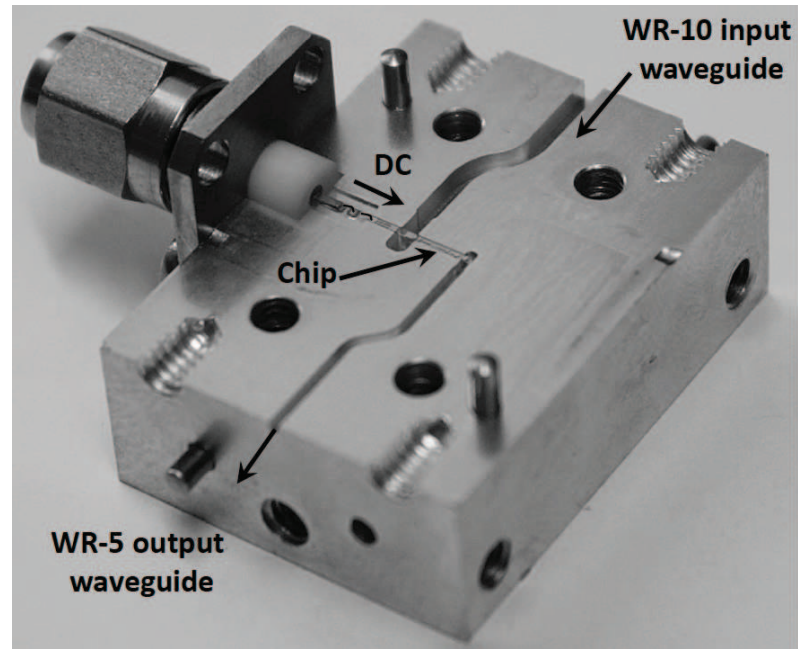

Fig. 3. A picture of the multiplier assembly showing input and output waveguide, SMA connector for DC biasing and location of the SuMA chip.

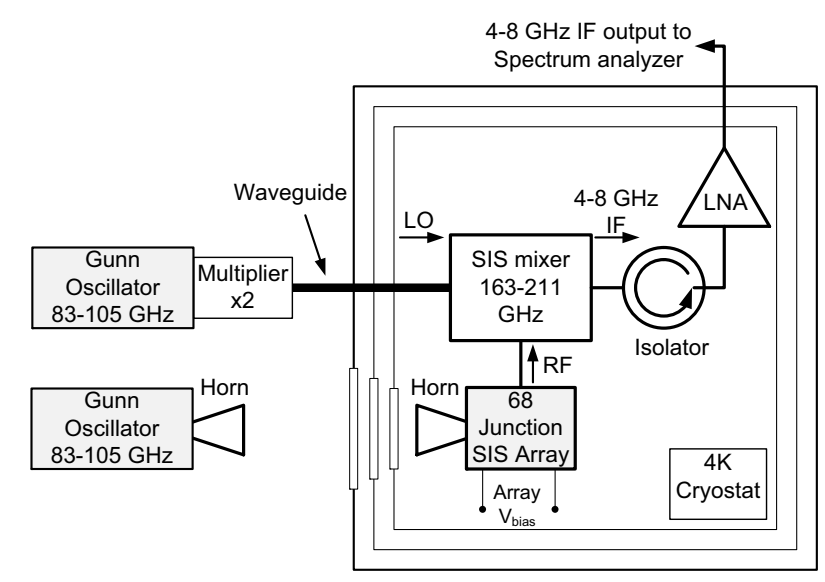

Fig. 4. Schematic of the experimental setup used to study the test device. The cryostat contains the SIS junction array connected directly to a $163-211 \mathrm{GHz}$ SIS mixer. Both the SIS mixer and the test array are pumped using two separate Gunn oscillators.

effect was carefully suppressed using the magnetic field. An isolator and a cryogenic low-noise amplifier were used at the output of the SIS mixer. The SIS mixer was pumped using a similar W-band Gunn oscillator and a frequency doubler [19] developed by the Rutherford Appleton Laboratory (RAL), UK. The IF output was analyzed with a spectrum analyzer.

From the DC I-V characteristics, the gap voltage and the normal state resistance $\left(R_{n}\right)$ of the whole array was found to be $195 \mathrm{mV}$ and $R_{n}=137 \Omega$ respectively. Which corresponds to $2.86 \mathrm{mV}$ gap voltage and $R_{n}=2.01 \Omega$ per junction, showing excellent agreement with the design value of $R_{n}=2 \Omega$.

One of the most important requirement on any frequency multiplier is that it preserves the spectral purity of the input signal. In order to investigate the spectral response of the SuMA, the LO frequency $\left(f_{L O}^{\text {mixer }}\right)$ of the SIS mixer used for this purpose was swept between $f_{L O}^{\text {mixer }} \rightarrow 171-203 \mathrm{GHz}$, and the SuMA was pumped with frequencies $\left(f_{S u M A}^{\text {in }}\right)$ such that,

$$
f_{S u M A}^{i n}=\frac{f_{L O}^{\text {mixer }} \pm(4 \rightarrow 8 G H z)}{2}
$$




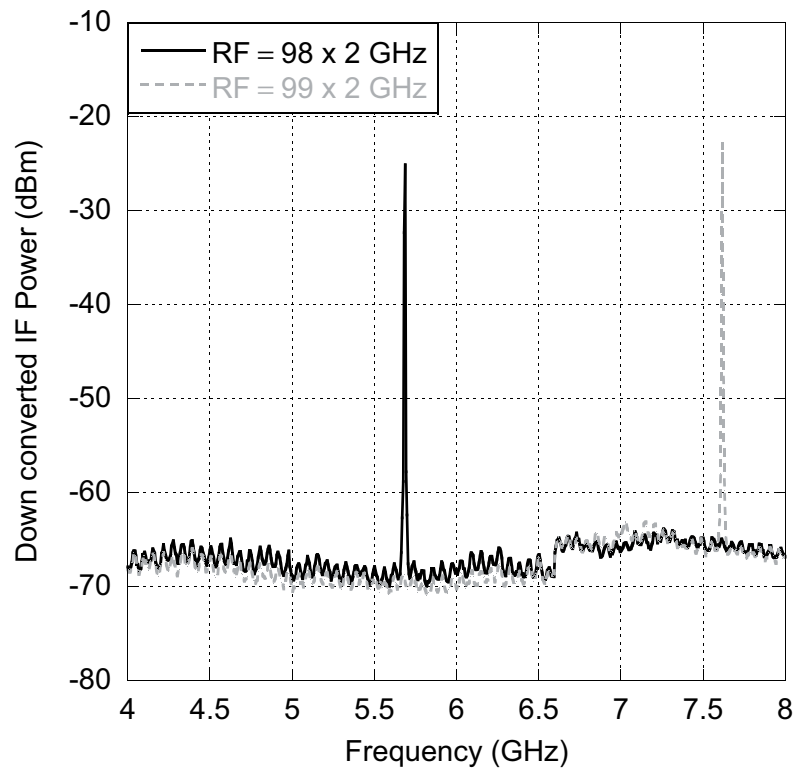

Fig. 5. Measured down converted output response of the SIS array. The SIS detector was pumped with a LO frequency of $\approx 190.3 \mathrm{GHz}$, and the SIS array was exited with a signal at $98 \mathrm{GHz}$ (solid black curve) and with 99 $\mathrm{GHz}$ (dashed gray curve).

in this way the expected $\times 2$ output from the multiplier would always fall inside the $4-8 \mathrm{GHz}$ band of the down converted mixer IF.

Fig. 5 shows the measured spectral response of the device, when the SuMA was pumped with a signal at $98 \mathrm{GHz}$, and at $99 \mathrm{GHz}$. In both the cases, the SIS mixer LO frequency was $\approx 190.3 \mathrm{GHz}$. We observed very strong frequency multiplication of the pumping signal by the SuMA. The solid black curve at $5.7 \mathrm{GHz}$ IF in Fig. 5 corresponds to a $98 \times 2$ $\mathrm{GHz}$ output frequency from the SIS multiplier array. Similarly, the dashed gray curve at $7.7 \mathrm{GHz}$ IF corresponds to output frequency of $99 \times 2 \mathrm{GHz}$. The observed signals were well above $45 \mathrm{~dB}$ from the noise floor, with no indication of any spurious signals. Measurements with several combination of the LO and RF frequencies were performed, all confirming frequency multiplication at second harmonics.

The dependence of frequency multiplication on the array bias voltage was investigated by fixing both the LO signal for the SIS mixer and the pumping signal for the SuMA, and measuring the IF power while sweeping the SuMA bias voltage. Fig. 6 show the measured power (solid black curve) of the detected $6 \mathrm{GHz}$ IF signal, when the LO frequency for the SIS detector mixer was set to $190 \mathrm{GHz}$, and the SuMA was pumped with $98 \mathrm{GHz}$ tone. The measured power of the multiplied second harmonic signal at $196 \mathrm{GHz}$ shows dependence on the array bias voltage. We observed multi-photo process in the SIS array response to the applied microwave excitation. The first peak below the gap voltage is believed to be associated with absorption of one photon whereas the second peak below the gap voltage corresponds to absorption of two photons at the pumping frequency. We also observed significant conversion of the input pumping signal into the second harmonic for DC bias above the gap voltage.

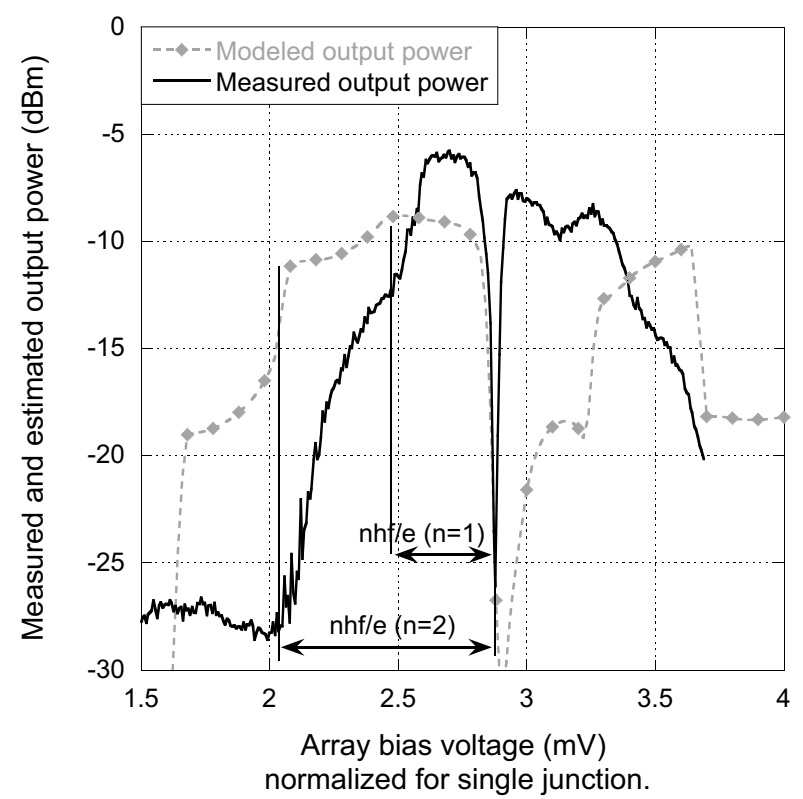

Fig. 6. Bias voltage dependence of the multiplied signal. With $190 \mathrm{GHz}$ LO frequency for the SIS mixer and while sweeping the SIS array bias voltage, the power of $196 \mathrm{GHz}$ signal was measured (solid black curve) at $6 \mathrm{GHz} \mathrm{IF}$ using spectrum analyzer. The voltage axis for the entire array is normalized for single junction. The dashed gray curve shows the estimated output power from the model.

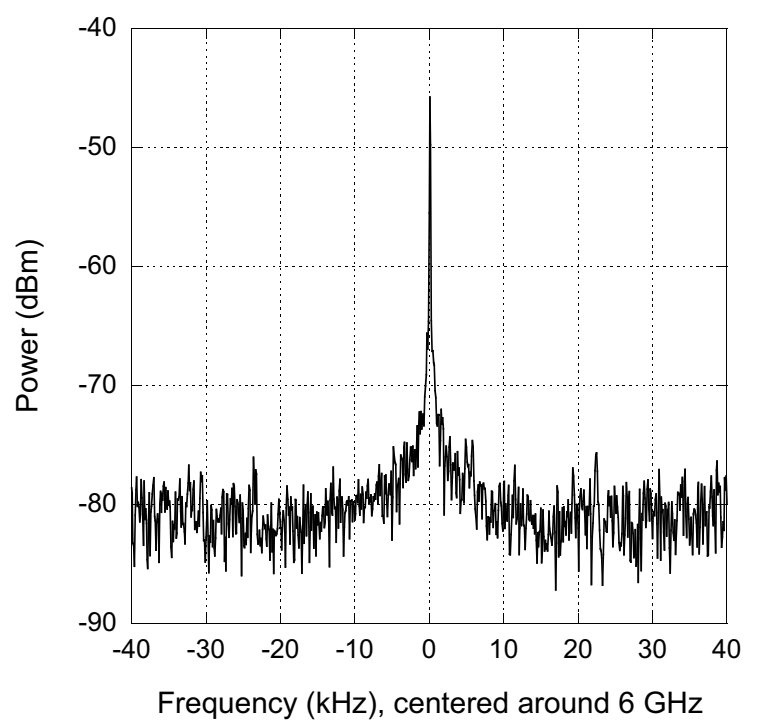

Fig. 7. Investigation of the line-width measurements of the multiplied signal. The spectra is centered at $6 \mathrm{GHz}$ with $80 \mathrm{kHz}$ span.

The LO sources used in radio astronomy e.g. Gunn oscillator, YIG etc., generally produce very sharp narrow-band tone. It is therefore necessary that the multipliers used in the subsequent LO chain preserves the spectral line-width of the original signal. Fig. 7 shows the measurement results to verify the line-width of the multiplied signal, using spectrum analyzer. In this measurement both the Gunn oscillators could not be phase locked due to unavailability of the phase lock loop (PLL) system. Therefore, the measurements were carried out using free running Gunn oscillators. Fig. 7 shows that 
the $-3 \mathrm{~dB}$ line-width of the output signal is better than the resolution bandwidth used for these measurements. The -30 $\mathrm{dB}$ line-width in this case was better than $2 \mathrm{kHz}$, even with free-running Gunn oscillators.

For comparison, we performed direct measurements of the Gunn oscillators spectrum and found very similar line-width of the pumping signal. This leads us to conclusion that the SuMA does not broaden the line-width of the multiplied signal noticeably.

\section{DiscusSION}

Accurate analytical modeling of the entire array would be a very challenging task. It would require a steady state solution of the large signal waveform across the entire array, and consideration of the distributed effects both at the pumping and the output frequencies would need to be taken into account.

In order to simulate array behavior and understand the measured results, a Matlab model was developed, with few simple but realistic assumptions. The Josephson effect is assumed to be completely suppressed by applied magnetic field. We assumed that the third and all higher harmonic of the LO are shorted by the array capacitance. We also assume that due to distributed nature of the array, the pumping power would decay along the array, such that the first junction in the array of 68 would be pumped harder than any next junction. This leads to the different pumping level of each individual junction in the distributed SIS multiplier array. The distributed effect is taken into account by adding the output power of the individual junctions with different phase. However, the DC bias voltage dependence of the power decay along the array for both, the pumping and the multiplied signal [9], is not addressed in the current model. The gray curve in Fig. 6 shows the estimated output power of the array, the estimated output power shows good resemblance with the measurements.

Since we use a series array, the second harmonic signal generated by the $68^{\text {th }}$ junction in the array has to propagate through 67 junctions before it can be extracted out. In this process some of the SIS junctions can get pumped at the second harmonic frequency. The effect of an SIS junction getting pumped at second harmonic has not been taken into account in our modeling.

In order to determine the maximum output power from the SuMA, measurements with various levels of input power and DC bias voltage were performed. The output power of the SIS array shows almost linear dependence with the input power up to a certain level, and then saturates.

Fig. 8 shows the effect of increasing the input power (pumping factor: $\alpha$ ). As the tunnel barrier is isolated from the substrate by two superconducting electrodes, which are good thermal isolators, at certain pumping level, the first few junctions in the array would be over-pumped, causing local heating inside the tunnel barrier. The degradation of the gap parameter $\delta V_{\text {gap }}$ in Fig. 8, is believed to be associated with the local heating of some of the junctions in the array, turning some junctions into normal mode.

This also confirms distributed nature of the array and the earlier assumption that the first junctions in the array are

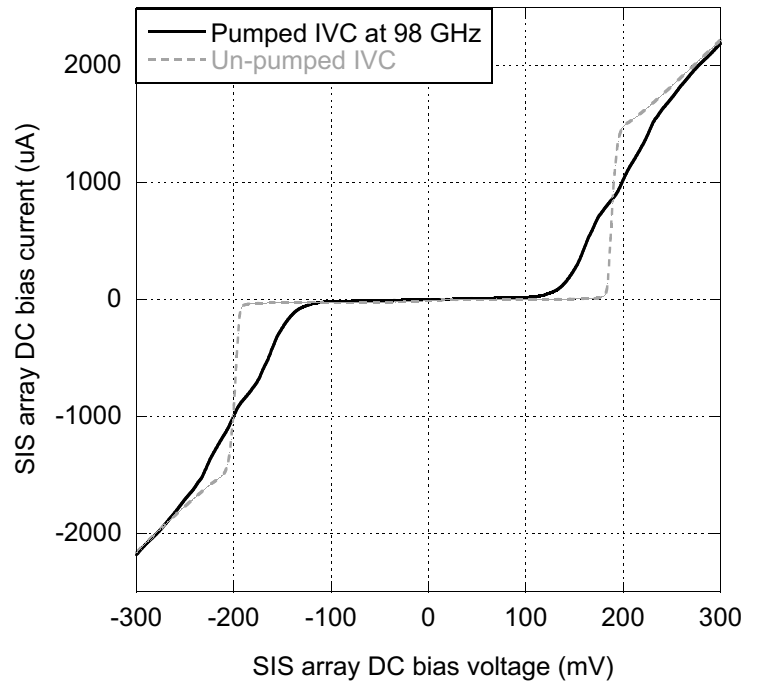

Fig. 8. Overheating of the SIS array at high level of applied pumping signal.

pumped harder than the others. At high values of $\alpha$, the first few junctions add a resistive component resulting in plot shown in Fig. 8. Further increase of the input power results in complete suppression of the gap feature, and the resulting I-V characteristic is just a straight line with slope $R_{n}$.

In order to asses if the SuMA could provide output power sufficient to pump a practical SIS mixer, the output from SuMA was directly connected to the LO port of the SIS mixer. Even though we were unable to see the pumped photon steps of the SIS mixer, we observed $10-20 \%$ increase in the SIS mixer sub-gap current. Estimation of the total output from the SuMA by taking out the total gain of the IF chain and mixer [10], gives about $\approx 8-10 n W$ at the WR-5 waveguide port of the multiplier.

\section{CONCLUSION}

We present results of the first experimental study of frequency multiplication in a distributed SIS multiplier array consisting of 68 SIS junctions. We experimentally observed off-chip generation of the second harmonic in $180-200 \mathrm{GHz}$ frequency band. The generated signal shows dependence on the DC bias voltage. The dependence exhibits features corresponding to single and multi-photon processes in the tunnel junction array. The measurements also confirmed no noticeable change in the spectral line-width of the multiplied signal as compared to the original pumping signal. The device modeling shows good resemblance with the measurements. The device, although far from providing enough power to pump a practical SIS mixer, may be considered as a first experimental step towards SIS frequency multipliers. Further development of the demonstrated principle of frequency multiplication may lead to a practical frequency multiplier device.

\section{ACKNOWLEDGMENT}

The authors would like to thank their colleagues at GARD Dr. Denis Meledin and Dr. Vincent Desmaris for their help 
in the fabrication process of the test devices, and Magnus Strandberg for assistance with data acquisition system. The authors would also like to thank Prof. Brian Ellison and Dr. Manju Henry, RAL, UK for providing the Schottky room temperature LO frequency doubler for the measurements.

\section{REFERENCES}

[1] A. Kerr and S.-K. Pan, "Some recent developments in the design of SIS mixers," International Journal of Infrared and Millimeter Waves, vol. 11, no. 10, pp. 1169-1187, 1990.

[2] K. Xiao, H. Ogawa, A. Mizuno, and Y. Fukui, "A $230 \mathrm{GHz}$ low noise subharmonically pumped SIS mixer," in IEEE MTT-S International Microwave Symposium Digest. 2000., vol. 1, 2000, pp. 573-576.

[3] V. Belitsky, S. Jacobsson, S. Kovtonjuk, E. Kollberg, and A. Ermakov, "100 GHz mixer vertically integrated (stacked) SIS junction array," International Journal of Infrared and Millimeter Waves, vol. 14, no. 5, pp. 949-957, 1993.

[4] V. Koshelets, A. Shchukin, S. Shitov, and L. Filippenko, "Superconducting millimeter wave oscillators and SIS mixers integrated on a chip," Applied Superconductivity, IEEE Transactions on, vol. 3, no. 1, pp. 2524-2527, 1993.

[5] D. Y. An et al., "Terahertz emission and detection both based on high- $\mathrm{T}_{c}$ superconductors: Towards an integrated receiver," Applied Physics Letters, vol. 102, no. 9, p. 092601, 2013. [Online]. Available: http://link.aip.org/link/?APL/102/092601/1

[6] O. Kiselev et al., "Balloon-borne superconducting integrated receiver for atmospheric research," Applied Superconductivity, IEEE Transactions on, vol. 21, no. 3, pp. 612-615, 2011.

[7] H. Foltz and J. Davis, "Calculated performance of SIS junctions as frequency multipliers," in IEEE MTT-S International Microwave Symposium Digest, 1990., vol. 1, May 1990, pp. 307-310.

[8] A. Kerr, "RF burnout power of SIS mixers," https://science.nrao. edu/facilities/alma/aboutALMA/Technology/ALMA_Memo_Series/ alma576/memo576.pdf, February 2008.

[9] J. R. Tucker and M. J. Feldman, "Quantum detection at millimeter wavelengths," Rev. Mod. Phys., vol. 57, no. 4, pp. 1055-1113, Oct 1985.

[10] B. Billade, A. Pavolotsky, and V. Belitsky, "A SIS mixer with $2 \mathrm{hf} / \mathrm{k}$ DSB noise temperature at $163-211 \mathrm{GHz}$ band," IEEE transaction on, Terahertz science and technology, vol. 3, no. 4, pp. 416-421, July 2013.

[11] A. Pavolotsky, D. Dochev, and V. Belitsky, "Aging- and annealinginduced variations in $\mathrm{Nb} / \mathrm{Al}-\mathrm{AlO} \mathrm{O}_{x} / \mathrm{Nb}$ tunnel junction properties," Journal of Applied Physics, vol. 109, no. 2, p. 024502, January 2011.

[12] V. Belitsky and M. Tarasov, "SIS junction reactance complete compensation," IEEE Transactions on Magnetics, vol. 27, no. 2, pp. 2638-2641, Mar 1991.

[13] V. Belitsky and E. Kolberg, "Superconductor-Insulator-Superconductor tunnel strip line features and applications," Journal of Applied Physics, vol. 80, no. 4, pp. 4741-4748, October 1996.

[14] EMDS, "Agilent, Electromagnetic Design System (EMDS)," "Attp://www.home.agilent.com/en/pc-1297137/ electromagnetic-design-system-emds?cc=SE\&lc $=$ swe.

[15] ADS, "Agilent, Advanced Design System (ADS)," http://www.home. agilent.com/en/pc-1297113/advanced-design-system-ads.

[16] J. Kooi et al., "A full height waveguide to thin film microstrip transition with exceptional RF bandwidth and coupling efficiency," Journal of Infrared and Millimeter Waves, vol. 24, no. 3, pp. 261-284, 2003.

[17] G. Yassin and S. Withington, "Analytical expression for the input impedance of a microstrip probe in waveguide," International Journal of Infrared and Millimeter Waves, vol. 17, no. 10, pp. 1685-1705, 1996.

[18] C. Risacher, V. Belitsky, V. Vassilev, and A. Pavolotsky, "A waveguide to microstrip transition with integrated Bias-T," IEEE microwave and wireless components letters, vol. 13, no. 7, pp. 262-264, July 2003.

[19] M. Henry et al., "Oscillator system development for the ALMA band 5 receiver," in Proceedings of the 4th UK/Europe-China Conference on Millimetre Waves and Terahertz Technologies, Glasgow, UK, Septemper 2011.

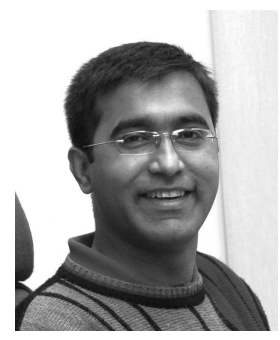

Bhushan Billade was born in India, in $1982 . \mathrm{He}$ received the B.E. degree in electronics and telecommunication engineering from the Aurangabad University, India, in 2003, and the M.Sc. in Radio astronomy and space sciences, and $\mathrm{Ph} . \mathrm{D}$. degree in electrical engineering from the Chalmers University of Technology, Gothenburg, Sweden, in 2005 and 2013, respectively. During his Ph.D., he worked on the development of low noise SIS receiver for the ALMA project. Currently, he is a staff member at the Onsala space observatory, Sweden.

In 2006, he was with the National Center for Radio Astrophysics, Pune, India. His research interests include microwave and millimeter wave MMIC, heterodyne receivers and mixers. His main focus is on low noise instrumentation for radio astronomy.

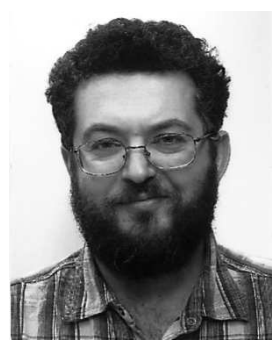

Alexey Pavolotsky received the M.S. and Ph.D. degrees from Moscow Aircraft Technology Institute/Technical University, in 1990 and 2003, both in material science and engineering. Since 2002, he is a Senior Research Engineer in the Group for Advanced Receiver Development, Onsala Space Observatory, Chalmers University of Technology, Gothenburg, Sweden.

His research interests include low-Tc thin film processing and characterization, as well as micro fabrication in general.

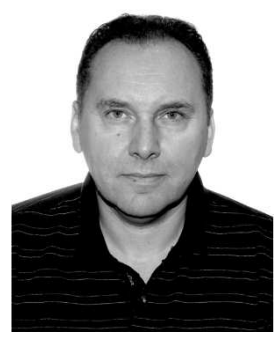

Victor Belitsky (M'95-SM'07) received the M.Sc. degree from the Moscow Telecommunication Institute, Moscow, Russia, in 1977, and the Ph.D. degree in experimental physics from the Institute of Radio Engineering and Electronics, USSR Academy of Sciences, Moscow, Russia, in 1990. He is currently a Professor and leader of the Group for Advanced Receiver Development (GARD) at the Department of Earth and Space Sciences, Chalmers University of Technology, Gothenburg, Sweden.

His research interests include THz electronics and components, instrumentation for radio astronomy and environmental science. 